\title{
Improving the Reference Frame by Radio-and Optical Astrometry of Radio Stars
}

\author{
Chr. de Vegt N. Zacharias \\ Hamburg Observatory, Germany \\ K.J. Johnston \\ Naval Research Laboratory, Washington D.C. USA \\ R. Hindsley \\ U.S.Naval Observatory, Washington D.C. USA
}

ABSTRACT. A longterm program of precise radio - and optical astrometry of selected radio stars has been conducted in the last decade by our observatories using the VLA and astrographs on both hemispheres. Positions of 54 stars north of -26 deg. declination including 6 MASER stars have been obtained. The program status and some results from the southern hemisphere are reported.

\section{Introduction}

The VLA has been used since the early ' 80 to determine precise astrometric parameters of selected radio stars in the primary extragalactic VLBI reference frame. For a recent detailed description of the observing program and previous results we refer to $/ 1 /$ and further references therein.

The main goal of the program is to provide a net of about 100 radio stars globally to link the present groundbased optical reference frame to the VLBI based extragalactic reference frame and to provide a similar link for the space based HIPPARCOS stellar net $/ 2 /$.

As most stars display radio emission at $\mathrm{cm}$ wavelengths only on the level of a few mJ, presently only the VLA can provide the necessary sensitivity although the accesible sky coverage is limited to $>-26$ deg. decl.

\section{Status of Radio Work}

At present precise positions ( $\pm 0.01-0.02$ arcsec) have been obtained for 54 stars, including 6 MASER stars. Radio proper motions ( $\pm 0.004 \mathrm{mas} / \mathrm{Yr}$ ) have been deterinined now for the stars HR1099 and UX Ari and second epoch observations have begun for additional stars. 
The quoted accuracies may be improved finally to the 1 mas level by incorporating additional calibrator sources in the close vicinity of the stars. In addition work on radio parallaxes has been started for UX Ari. The diagram displays the distribution of the present radio stars. The lack of observations on the southern hemisphere is obvious, however, the coverage extends already far enough to the south to allow for a rotation solution in the comparision of the reference frames.

\section{Status of Optical Observations}

Almost all radio stars of the present sample are optically brighter than visual magnitude 12 . Therefore they can be tied to the optical fundamental reference frame as given by the IRS-catalog easily by use of high quality wide field astrographs on both hemispheres.

Due to the favourable field size of these instruments which is at least $25 \mathrm{sq} . \mathrm{deg}$. and corresponding number of IRSreference stars, the positions of the radio star can be determined with high systematic accuray $(<0.05$ arcsec $)$ in the FK5 system. Table 1 summarizes the main instrumental parameters.

Table 1. Main Instrumental Parameters of Astrographs

\begin{tabular}{|c|c|}
\hline Northern Hemisphere & Southern Hemisphere \\
\hline Site: Hamburg +53.5 Lat. & New Zealand -41.8 Lat. \\
\hline Aperture: $23 \mathrm{~cm}$ & $20 \mathrm{~cm}$ \\
\hline Scale: $100 " / \mathrm{mm}$ & $100 " / \mathrm{mmm}$ \\
\hline Field: $6 \times 6$ deg. & $5 \times 5$ deg. \\
\hline Spectral range: $5200-5800 \AA$ & $5000-5800 \AA$ \\
\hline Gratings: 4 mag / 6 mag & $4 \mathrm{mag} / 6 \mathrm{mag}$ \\
\hline Emulsion + Filter: $103 a G+O G 515$ & $103 a G+G G 495$ \\
\hline Plate size: $240 \times 240 \times 6.4 \mathrm{~mm}$ III & $\begin{array}{c}203 \times 254 \times 6.4 \mathrm{~mm} \text { III } \\
\text { III }=(\mathrm{micro} \text { (I) })\end{array}$ \\
\hline Reference star Catalog: AGK3RN & SRS **) \\
\hline
\end{tabular}

*) USNO Southern Hem. Station at Black Birch Astron. Observatory

**) SRS=final Version USNO /3/, both cat. FK4/B1950 and FK5/J2000 version. 
Normally 4 plates (single exposure) or 2 plates (double exp.) have been used for each object and 2 (1) plates have been combined in pairs, exposed symmetrical to the meridian and in opposite orientation of the telescope to the sky to minimize possible residual color dependent effects of the optics and atmosphere /4/. Depending on the maynitude of the radio star, a 6 or 4 mag. grating has been used. Most of the plates have been measured on the recently completely automated and CCD-camera based MANN-Comparator of Hamburg Observatory /5/. Typical mean errors of 0.05 arcsec for the optical positions have been achieved. Both the AGK3RN and SRS catalogs have been transfered to the FK5-system using the analytical expressions, as developed at ARI-Heidelberg /6/. As the systematic differences FK5-FK4 reach their largest amplitudes on the southern hemisphere, radio stars may provide an excellent test, at least locally. Table 2 shows some examples. The m.e. of a single "O-R" difference is 0.05 arcsec wich demonstrates the systematic quality of the SRS catalog obviously. Similar results have been obtained recently for a small sample of extragalactic radio sources $/ 7 /$.

Table 2. Examples of Southern Hemisphere System Comparisons "Optical - "Radio" (SRS cat.FK5/J2000)

$\begin{array}{lll}\text { Star } & \text { DA*cos }(\text { dec }) & \text { DD } \\ \text { TW Lep } & +0.05 & +0.04 \\ \text { RV Lib } & +0.11 & -0.17 \\ \text { HU Vir } & -0.06 & -0.07\end{array}$

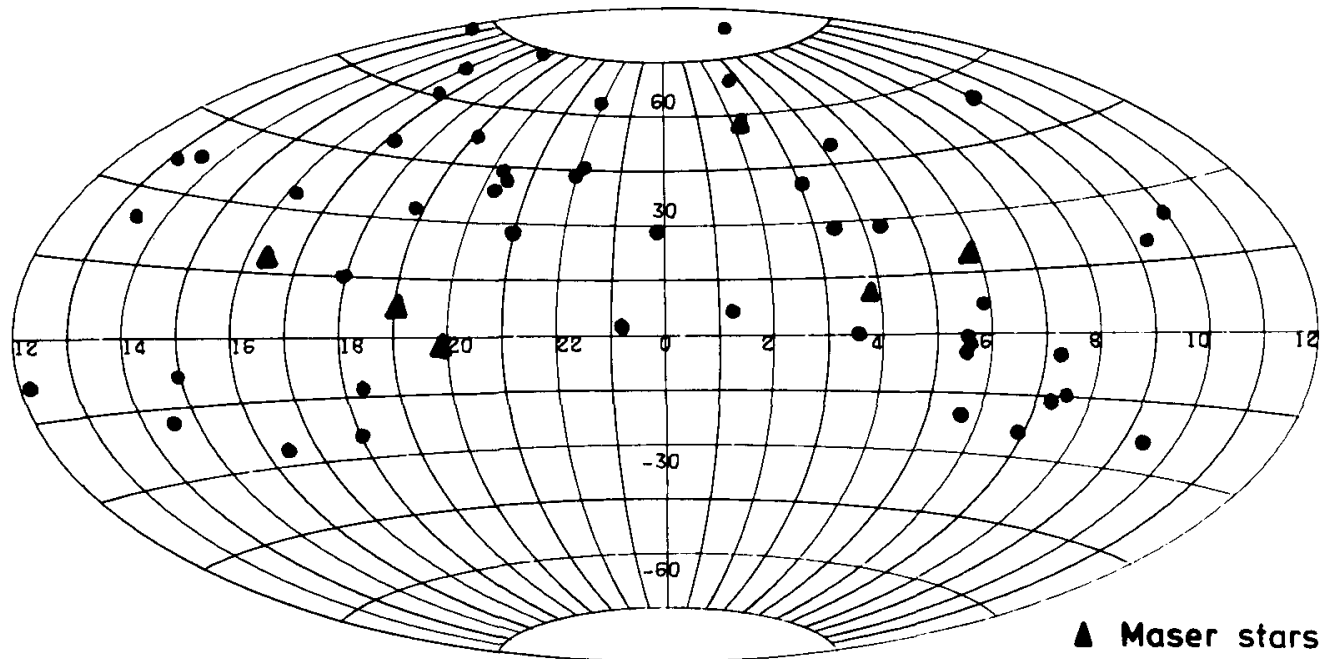

Distribution of stars from VLA-program 


\section{Acknowledgements}

CdV acknowledges financial support from the Bundesministerium für Forschung und Technologie (BMFT) under grant no. 1000018-3 (Hipparcos)

\section{References}

1. de Vegt Chr.,Johnston K.J.,Russell J.,Hughes J.A., Jauncey D.,Nicolson G.: 1988, Proc.Hipparcos Coll. Prep. of the Input Cat.II, p.469

2. de Vegt Chr.,Zacharias N.,Johnston K.J.: 1991, Proc. 28 th. COSPAR 1990, in press

3. Smith C.A.,Corbin T...E.,Hughes J.E., Jackson E.S., Khrutskaya E.V. ,Poloshentsev A.D., Poloshentsev D.D., Yagudin L.I., Zverev M.S.: 1990, Proc.IAU Symposium 141, p. 457

4. de Vegt Chr.: 1991, Proc. IAU Coll.100, Astrophys.Space Science, in press

5. Winter L.: 1989, Diplomarbeit, Universität Hamburg

6. Schwan H.: 1989, private communication

7. Russell J.,Johnston K.J., Jauncey D., White G., Reynolds J., Harvey B., Nothnagel A., Nicolson G.,Kingham K., Ma C., de Vegt Chr., Hindley R. ,Zacharias N.: 1991, A RadioOptical Reference Frame, paper III, submitted to A.J. 